\title{
NIVELES DE ESTRÉS ACADÉMICO Y SU RELACIÓN CON RESPUESTAS ORGÁNICAS EN ESTUDIANTES DEL I SEMESTRE DE LA FEN*
}

\author{
Jaime P., Norma ${ }^{1}$ \\ Facultad de Enfermería de la Universidad Nacional del Centro del Perú
}

\begin{abstract}
RESUMEN
El objetivo del presente trabajo fue identificar y determinar los niveles de estrés académico que provocan las situaciones generadoras de estrés, la frecuencia de ocurrencia de las respuestas referidas a los tres niveles de respuesta del organismo del estudiante y la relación existente entre cada una de las situaciones generadoras de estrés y las respuestas en los diferentes niveles manifestadas por los estudiantes del I semestre de la FEN 2006-I. El estudio fue observacional, descriptivo y el área de trabajo la Facultad de Enfermería, Población: el total de estudiantes del primer semestre académico 2006-I (24 estudiantes) las técnicas y procedimientos de recolección de datos fueron: la encuesta directa. La información, recogida se procedió a analizar e interpretar siguiendo un proceso manual y utilización de la estadística descriptiva. Posteriormente se utilizó para el vaciado de datos al programa Estadístico SPSS. En esta fase del estudio se obtiene como resultado que la clasificación por niveles de estrés se encuentra en: alto, medio y bajo y la frecuencia de ocurrencia de las respuestas referidas a los tres niveles de respuestas del organismo del estudiante de acuerdo al padecimiento es: casi siempre, con bastante frecuencia y algunas veces y la relación entre cada situación generadora de estrés con cada ítem de los tres niveles de respuesta analizada mas específicamente es: los ítemes 2,4,6,10 y 11 se relaciona con las manifestaciones cognitivas, fisiológicas y motoras de una forma significativa y altamente significativa, mientras que el ítem 1 se relaciona significativamente con las manifestaciones solo cognitivas y fisiológicas, los ítemes 5 y 8 se relaciona significativamente solo con las manifestaciones cognitivas y el ítem 9 se relaciona únicamente con las manifestaciones motoras, finalmente los ítemes 3 y 7 no se relacionan con ninguna lo que podemos sintetizar en que si bien es cierto los 11 ítemes se comportan como situaciones generadoras de estrés por los resultados obtenidos dos de ellos no guardan relación con ninguna de las respuestas o manifestaciones frente a las situaciones de estrés por parte de los estudiantes. La vulnerabilidad al estrés es un fenómeno de elevada frecuencia en los estudiantes del primer semestre de la facultad de enfermería, el estrés percibido para cada situación en el presente estudio es, en líneas generales, elevado, la mayoría de los estudiantes se encuentran con alto y medio nivel de estrés y un grupo pequeño con bajo estrés, la frecuencia de ocurrencia de las respuestas referidas a los tres niveles de respuesta del organismo del estudiante es siempre, casi siempre y algunas veces y su relación por ítem de las situaciones generadoras de estrés es en la mayoría de ellos directa significativa $(p<0,05)$ y altamente significativa $(p<0,01)$ con los diferentes niveles de respuestas frente a ella los que pueden deteriorar la salud si no se establecen mecanismos de afrontamiento que esté respaldado por la facultad a través de políticas de prevención y promoción de la salud mental del estudiante.
\end{abstract}

Palabras clave: estrés, niveles de respuestas, fisiológico, cognitivo, motor, afrontamiento, pensamiento negativos. 


\title{
LEVELS OF ACADEMIC STRESS AND ITS RELATION BETWEEN ORGANIC REACTIONS IN STUDENTS OF THE FIRST CYCLE FROM NURSING FACULTY
}

\begin{abstract}
The objective of the present investigation was to identify and determine high levels of academic stress which provoke situation the facilitate the stress the frequency of incidence of the answers referring to the three levels of answers in the students organisme and the relationship among each of causing situations of stress and the first cyde students studio and the working area was the nursing faculty which population is the whole number of students who belong to the first cyde of 2006 - I period (24 students), and the techniques and procedure of collecting information were: the direct survey information it was analyzed and interpreted following a manual process and the use of descriptive statistics; lateron the SPPS statistical program was urea in order to process this information. In this of the study the stress classification was levels: high, intermediate and low and the frequent of imminent of the answer refereed to the three levels of answers in the students organisms according to the physiological reactions is almost always with lost of frequency and sometimes the relationship among each situation promets the stress with each item and the anodized answers of the three levels more specifically are: the items 2,4,6,10 and 11 are in relations directly with the cognitive physicologuol and motor manifestations are highly significative while item 1 is just significativels in relation with the pliysiological and cognitive manifestational the item 5 and 8 are in significant related the just motor manifestations. Finally the items 3 and 7 don't have any relationship among then motor manifestations; finally the items 3 and 7 don't have any relationship among them. From this are can say that even though the 11 items are situations that cause stress and according to the results obtained 2 out of then don't have relationship with any of the answers on manifestation in front of the situations that cause stress in the students the vulnerability of stress is a phenomenon of high frequency in the student of the semester of the nursing faculty. The stress index for each situation in this study is in general words, high. The majority of the students are in levels high and intermediate and a very small group with a low index, for each situation in this study is in general words high the majority of the students are in levels high and intermediate and a very small groop with a low index of stress the frequency of incidence of these answers seferred the three levels of answers in the students organisms are always almost always and sometimes and its relationship by items of the situations causing stress are in most of the cases dereatty signifificative $(p<0.05)$ and highly significative $(p<0.01)$ with the different $y$ levels of answers and in front of theme the situations that con deteriorate the students health if they don't settle deacon ways to control this risk and should be supported by the nursing faculty through programs to present and promote the mental health of the students.
\end{abstract}

Key Words: Stress, Levels of answer, physiological, cognitive, motor, confrontation, negative thoughts.

* Este trabajo de investigación fue recibido el 20/11/2007, retornado para su revisión el 22/09/2008 y aprobado para su publicación el 27/02/2009.

1. E-mail: norjaimep@yahoo.es 


\section{INTRODUCCIÓN}

Corroborado está que en el I semestre de la FEN, los estudiantes que la cursan manifiestan estrés ante situaciones académicas que enfrentan, repercutiendo este estrés en manifestaciones tanto fisiológicas, cognitivas y motoras. Por tanto para la proyección de su abordaje fue necesario saber primero: los niveles de estrés en los que estarían ubicados las distintas situaciones académicas generadoras de estrés, segundo: la frecuencia de ocurrencia de las respuestas referidas a los tres niveles de respuesta del organismo del estudiante y tercero cómo cada situación generadora de estrés se relaciona con cada respuesta ya sea fisiológica, cognitiva como motora manifestada por los estudiantes. Existen estudios relacionados a estrés en general sin embargo a través del arqueo de fuentes, a nivel internacional se pudo ubicar algunas relacionadas al ámbito Universitario las que por su importancia y contribución al trabajo se mencionan.

Entre ellas las de Marty M.; Matías Lavín, G.; Maximiliano Figueroa M.; Larraín de la C., Demetrio y Cruz M., Carlos de la Facultad de Medicina, Universidad de los Andes, Santiago de Chile quienes realizaron una investigación sobre Prevalencia de estrés en estudiantes del área de la salud y su relación con enfermedades infecciosas, el estudio se realizó con alumnos de medicina de la Universidad de los Andes, Chile: se estableció en este trabajo que un importante porcentaje de estudiantes presentan estrés sintomático durante un período de mayor exigencia académica, especialmente las mujeres y en los primeros años de las carreras. Lo que ameritaba la intervención inmediata dado que el estado de estrés en los años siguientes de estudio y en los años profesionales puede llevar a serias consecuencias psicosociales. La etiología de la enfermedad es multifactorial, pues depende de la interacción de mente y cuerpo, y de un ambiente psicosocial y académico que ejerce gran influencia. Pellicer, Salvador y Benet, al estudiar el problema del estrés en estudiantes de Medicina de Bogotá expresan que Indiscutiblemente el estrés afecta notoriamente el desempeño académico de los estudiantes de primero a quinto semestre de medicina, siendo mas notorio en tercero y quinto semestres en virtud a su mayor carga académica. Los estresores a los que están expuestos los estudiantes de medicina son: excesiva carga académica, currículo muy apretado, horario muy extenso, situación económica precaria, choque cultural de las personas procedentes de fuera de Bogotá, y en menor grado desordenes sentimentales.

Con el desarrollo de este trabajo se pretendió llegar a un conocimiento más exhaustivo del estrés académico que padecen los estudiantes del primer semestre de la Facultad de Enfermería 2006-I, tanto en lo que tiene que ver con las situaciones específicas que favorecen su aparición como en las reacciones que dichas situaciones provocan en los individuos. No se planteó ninguna hipótesis porque el trabajo fue inminentemente descriptivo. El objetivo fue determinar los niveles de estrés académico que provocan las situaciones generadoras de estrés, la frecuencia de ocurrencia de las respuestas referidas a los tres niveles de respuesta del organismo del estudiante y la relación existente entre cada una de las situaciones generadoras de estrés y las respuestas manifestadas por los estudiantes del I semestre de la FEN 2006-I, de tal modo que nos permita buscar mecanismos de afrontamiento y fomentar una vida estudiantil más saludable en el futuro.

\section{MATERIAL Y MÉTODOS}

Los sujetos que participaron en esta investigación fueron los estudiantes del primer semestre académico de la Facultad de Enfermería en un número de 24 estudiantes (total) matriculados. El estudio fue observacional, descriptivo. El área de estudio: fue la Facultad de Enfermería. y la población: el total de estudiantes del primer semestre académico 2006-I de la Facultad de Enfermería. las técnicas y procedimientos de recolección de datos para la recolección de información fueron: utilización de la investigación bibliográfica de fuente secundaria es decir a través de los textos y de la internet en un primer momento, aplicando la técnica del fichaje en sus diferentes variantes. En un segundo momento las técnicas y procedimientos de recolección de datos fueron: la información de la encuesta directa, la que utilizó como instrumentos el cuestionario autoaplicativo, en número de dos de la primera fase de investigación es decir el Inventario de Estrés Académico (I.E.A.), este inventario que incluyó once situaciones potencialmente generadoras de estrés en los estudiantes dentro del ámbito académico e 
inventario de tipo de respuestas (ITR) que incluyó 12 tipos de respuesta de los estudiantes frente al estrés, clasificándose por niveles de estrés académico y la frecuencia de ocurrencia de las respuestas referida a los tres niveles de respuesta del organismo para luego analizar la relación entre cada una de las situaciones generadoras de estrés y las respuestas manifestadas por los estudiantes del I semestre de la FEN 2006-I.

Para cada una de las situaciones planteadas se presentaron una escala con valores de 1 a 5 (donde 1 representó nada de estrés y 5 mucho estrés), que indicaron el nivel de estrés, que dicha situación pudo generar y el segundo instrumento inventario de tipo de respuestas (ITR) incluyó 12 tipos de respuesta de los estudiantes frente al estrés donde se recogió información en torno a los tres niveles de respuesta, fisiológico, cognitivo y motor, que el organismo experimenta cuando se ve expuesto a situaciones de estrés.

La frecuencia de ocurrencia de las respuestas, referidas a los tres niveles de respuesta del organismo, se evaluó en una escala de 1 a 5, donde 1 significó casi nunca o nunca y 5 significó casi siempre o siempre. La distribución de los elementos quedó conformada de la siguiente forma: nivel fisiológico 4 elementos $(2,5$, 8 y 11), nivel cognitivo 5 elementos $(1,4,7,10$ y 12) y nivel motor 3 elementos $(3,6$ y 9$)$. Las técnicas de procesamiento y análisis de datos que se utilizaron fue: de la estadística descriptiva y posteriormente se utilizó para el vaciado de datos al programa estadístico SPSS. y luego se procedió con la interpretación de los resultados.

Materiales y equipos a utilizados fueron de escritorio varios y equipos como computadora y paquete estadístico SPSS.

\section{RESULTADOS}

La vulnerabilidad al estrés es un fenómeno de elevada frecuencia en los estudiantes del I semestre de la FEN 2006-I ya que el estrés percibido para cada situación en el presente estudio es, en líneas generales, elevado. El puntaje de situaciones potencialmente generadoras de estrés académico analizado globalmente oscila entre 18 y 43 , de un rango esperado entre 11 y 55, lo que indica claramente el comportamiento de estas situaciones como estresores. Respecto al tipo de respuestas frente al estrés académico también enfocado en forma general, el puntaje de respuesta frente al estrés académico fluctúa entre 17 y 50 , de un rango esperado entre 12 y 60 lo que significa su clara relación con las situaciones generadoras de estrés académico.

La relación entre el puntaje de situación generadora de estrés académico y el puntaje de respuesta frente al estrés académico es directa, creciente o positiva, lo que indica que a medida que aumenta el puntaje de situación generadora de estrés también aumenta el puntaje de respuesta. Se intuye, entonces, una correlación positiva entre estas puntuaciones, tal como se aprecia en el valor del coeficiente de correlación lineal de Pearson. Dicho coeficiente es 0,7556 , un coeficiente positivo y significativo, al mostrar un $p$ valor de 0 , menor de 0,05 .

Los coeficientes de correlación corroboran la relación positiva existente entre las puntuaciones de situación generadora de estrés académico y de las diferentes manifestaciones fisiológicas, cognitivas y motoras. Estas afirmaciones, se dan con mayor intensidad entre situación generadora de estrés y manifestaciones cognitivas que alcanza una correlación de 0,7441 y con menor intensidad entre situación generadora y manifestaciones motoras que logra una correlación de 0,6349 . Por tanto si aumentan el grado de estrés en los estudiantes se acentuará las respuestas cognitivas en primera instancia en sus más altas frecuencias así como también sucederá el mismo fenómeno en los otros niveles (fisiológico y motor).

Aun cuándo éstas sean en menor intensidad respecto a la primera. Los estresores más resaltantes a los que están expuestos los estudiantes de enfermería son la falta de tiempo para poder cumplir con las actividades académicas (excesiva carga académica) realización de exámenes, realización de trabajos obligatorios para aprobar las asignaturas, exposición de trabajos en clase y en menor grado trabajar en equipo o grupo. De acuerdo a un análisis más específico, los niveles de estrés presentes en los estudiantes del I semestre de la Facultad de Enfermería 2006-I son altos, categorizado por la intensidad padecida por los estudiantes es decir mucho y bastante estrés; medio representado por poco estrés y nivel bajo representado por algo de estrés o nada de estrés. 
Cuadro 1. Niveles de estrés que padecen los estudiantes provocado por situaciones generadoras de estrés y la frecuencia de reacciones referidas a los tres niveles de respuesta del organismo es casi siempre, con bastante frecuencia y algunas veces.

\begin{tabular}{|c|c|c|c|}
\hline $\begin{array}{l}\text { NIVELES DE } \\
\text { ESTRÉS }\end{array}$ & $\begin{array}{l}\text { NÚMERO DE } \\
\text { ESTUDIANTES }\end{array}$ & SITUACIÓN GENERADORA DE ESTRÉS & $\begin{array}{l}\text { ORDEN DE } \\
\text { IMPORTANCIA }\end{array}$ \\
\hline \multirow{8}{*}{ (bastante y mucho estrés) } & 16 & \multirow{4}{*}{$\begin{array}{l}\text { Falta de tiempo para poder cumplir con las actividades } \\
\text { académicas. } \\
\text { Realización de un examen. } \\
\text { Sobrecarga académica (exceso } \mathrm{N}^{\circ} \text { de créditos llevados). } \\
\text { Realización de trabajos obligatorios para aprobar la } \\
\text { asignatura (búsqueda de material necesario, redactar el } \\
\text { trabajo, etc.). }\end{array}$} & Primer lugar \\
\hline & 13 & & Segundo lugar \\
\hline & 11 & & Tercer lugar \\
\hline & 10 & & Cuarto lugar \\
\hline & 7 & Exposición de trabajos en clase. & Quinto lugar \\
\hline & 7 & Competitividad entre compañeros. & Sexto lugar \\
\hline & 4 & La tarea de estudio. & Séptimo lugar \\
\hline & 4 & Trabajo en grupo. & Octavo lugar \\
\hline $\begin{array}{l}\text { GRADO DE } \\
\text { ESTRÉS }\end{array}$ & $\begin{array}{l}\text { NÚMERO DE } \\
\text { ESTUDIANTES }\end{array}$ & SITUACIÓN GENERADORA DE ESTRÉS & $\begin{array}{l}\text { ORDEN DE } \\
\text { IMPORTANCIA }\end{array}$ \\
\hline \multirow{6}{*}{ MEDIO } & 10 & \multirow{5}{*}{$\begin{array}{l}\text { Exposición de trabajos en clase. } \\
\text { Falta de tiempo para cumplir con las actividades } \\
\text { académicas. } \\
\text { Realización de un examen. } \\
\text { Sobrecarga académica. } \\
\text { Intervenciones en el aul a (responder a una pregunta del } \\
\text { profesor, realizar preguntas, participar en coloquios). }\end{array}$} & Primer lugar \\
\hline & 8 & & Segundo lugar \\
\hline & 8 & & Tercer lugar \\
\hline & 8 & & Cuarto lugar \\
\hline & 7 & & Quinto lugar \\
\hline & 7 & La tarea de estudio. & Sexto lugar \\
\hline \multirow[t]{5}{*}{ (poco estrés) } & 5 & \multirow{5}{*}{$\begin{array}{l}\text { Competitividad entre compañeros. } \\
\text { Realización de trabajos obligatorios para aprobar la } \\
\text { asignatura. } \\
\text { Trabajos en grupo. } \\
\text { Subir al despacho del profesor en horas de tutoría. } \\
\text { Masificación de aulas. }\end{array}$} & Séptimo lugar \\
\hline & 4 & & Octavo lugar \\
\hline & 4 & & Noveno lugar \\
\hline & 3 & & Décimo lugar \\
\hline & 1 & & Décimoprimer lugar \\
\hline $\begin{array}{l}\text { GRADO DE } \\
\text { ESTRÉS }\end{array}$ & $\begin{array}{l}\text { NÚMERO DE } \\
\text { ESTUDIANTES }\end{array}$ & SITUACIÓN GENERADORA DE ESTRÉS & $\begin{array}{c}\text { ORDEN DE } \\
\text { IMPORTANCIA }\end{array}$ \\
\hline \multirow{10}{*}{$\begin{array}{l}\text { BAJO } \\
\text { (algo de estrés) }\end{array}$} & 11 & $\begin{array}{l}\text { Intervenciones en el aula (responder a una pregunta del } \\
\text { profesor, realizar preguntas, participar en coloquios). } \\
\text { Masificación de las aulas. }\end{array}$ & Primer lugar \\
\hline & 10 & La tarea de estudio. & Segundo lugar \\
\hline & 10 & Trabajo en grupo. & Tercer lugar \\
\hline & 9 & Subir al despacho del profesor en horas de tutoría. & Cuarto lugar \\
\hline & 8 & Competitividad entre compañeros. & Quinto lugar \\
\hline & 7 & \multirow{5}{*}{$\begin{array}{l}\text { Exposición de trabajos en clase. } \\
\text { Realización de trabajos obligatorios para aprobar la } \\
\text { asignatura. } \\
\text { Sobrecarga académica. } \\
\text { Realización de un examen. } \\
\text { Masificación de las aulas. }\end{array}$} & Sexto lugar \\
\hline & 5 & & Séptimo lugar \\
\hline & 4 & & Octavo lugar \\
\hline & 3 & & Noveno lugar \\
\hline & 3 & & Décimo lugar \\
\hline \multirow[t]{4}{*}{$\begin{array}{l}\text { GRADO DE } \\
\text { ESTRÉS }\end{array}$} & $\begin{array}{l}\text { NÚMERO DE } \\
\text { ESTUDIANTES }\end{array}$ & SITUACIÓN GENERADORA DE ESTRÉS & $\begin{array}{c}\text { ORDEN DE } \\
\text { IMPORTANCIA }\end{array}$ \\
\hline & 20 & Masificación de las aulas. & Primer lugar \\
\hline & 12 & $\begin{array}{l}\text { Subir al despacho del profesor en horas de tutoria } \\
\text { La tarea de estudio. }\end{array}$ & Segundo lugar \\
\hline & 6 & Trabajo en grupo. & Tercer lugar \\
\hline AUSENCIA & 5 & $\begin{array}{l}\text { Intervenciones en el aula (responder a una pregunta del } \\
\text { profesor realizar prequntas participar en cologuios) }\end{array}$ & Cuarto lugar \\
\hline DE ESTRÉS & 5 & Masificación de las aulas. & Quinto lugar \\
\hline \multirow[t]{4}{*}{ (Nada de estrés) } & 5 & $\begin{array}{l}\text { Realización de trabajos obligatorios para aprobar la } \\
\text { asignatura. }\end{array}$ & Sexto lugar \\
\hline & 4 & Competitividad entre compañeros & Séptimo lugar \\
\hline & 3 & La tarea de estudio. & Octavo lugar \\
\hline & 1 & $\begin{array}{l}\text { Sobrecarga académica (exceso número de créditos } \\
\text { llevados). }\end{array}$ & Noveno lugar \\
\hline
\end{tabular}


Cuadro 2. Frecuencia de padecimiento por niveles de respuesta frente a situaciones provocadoras de estrés (tipos de respuesta por cada situación de estrés).

\begin{tabular}{|c|c|c|c|c|}
\hline $\begin{array}{l}\text { Frecuencia de } \\
\text { padecimiento }\end{array}$ & $\begin{array}{c}\text { Niveles de respuesta frente } \\
\text { a situaciones provocadoras } \\
\text { de estrés }\end{array}$ & Ítemes de cada nivel & $\begin{array}{c}\text { Número } \\
\text { de estudiantes }\end{array}$ & $\begin{array}{c}\text { Orden } \\
\text { de } \\
\text { importancia } \\
\end{array}$ \\
\hline \multirow{3}{*}{ CASI SIEMPRE } & Nivel cognitivo & $\begin{array}{l}\text { Me preocupo. } \\
\text { Siento miedo. } \\
\text { - Tengo pensamientos o sentimientos negativos. } \\
\text { - Me siento inseguro de mi mismo. } \\
\text { - Siento ganas de llorar. }\end{array}$ & $\begin{array}{l}12 \\
5 \\
- \\
2 \\
2\end{array}$ & Primer lugar \\
\hline & Nivel fisiológico & $\begin{array}{l}\text { - El corazón me late muy rápido y/o me falta el } \\
\text { aire y la respiración es agitada. } \\
\text { - Siento molestias en el estómago. } \\
\text { - Me tiemblan las manos o las piernas. } \\
\text {-Se me seca la boca y tengo dificultades para } \\
\text { tragar. }\end{array}$ & $\begin{array}{l}2 \\
5 \\
3 \\
2\end{array}$ & Segundo lugar \\
\hline & Nivel motor & $\begin{array}{l}\text { - Realizo movimientos repetitivos con alguna } \\
\text { parte de mi cuerpo. } \\
\text { - Fumo, como o bebo demasiado. } \\
\text { - Me cuesta expresarme verbalmente o a veces } \\
\text { tartamudeo. }\end{array}$ & $\begin{array}{l}2 \\
- \\
4\end{array}$ & Tercer lugar \\
\hline \multirow{3}{*}{$\begin{array}{l}\text { CON BASTANTE } \\
\text { FRECUENCIA }\end{array}$} & Nivel cognitivo & $\begin{array}{l}\text { Me preocupo. } \\
\text {-Siento miedo. } \\
\text { - Tengo pensamientos o sentimientos negativos. } \\
\text { - Me siento inseguro. } \\
\text { - Siento ganas de llorar. }\end{array}$ & $\begin{array}{l}5 \\
1 \\
- \\
3 \\
3\end{array}$ & Primer lugar \\
\hline & Nivel fisiológico & $\begin{array}{l}\text { - El corazón me late muy rápido y/o me falta el } \\
\text { aire y la respiración es agitada. } \\
\text { - Siento molestias en el estómago. } \\
\text { - Me tiemblan las manos o las piernas. } \\
\text { - Se me seca la boca y tengo dificultades para } \\
\text { trazar. }\end{array}$ & $\begin{array}{l}3 \\
7 \\
1 \\
1\end{array}$ & Segundo lugar \\
\hline & Nivel motor & $\begin{array}{l}\text { - Realizo movimientos repetitivos con alguna } \\
\text { parte de mi cuerpo, me quedo paralizado o mis } \\
\text { movimientos son torpes. } \\
\text { - Fumo, como o bebo demasiado. } \\
\text { - Me cuesta expresarme verbalmente o a veces } \\
\text { tartamudeo. }\end{array}$ & $\begin{array}{l}- \\
1\end{array}$ & Tercer lugar \\
\hline \multirow{3}{*}{ ALGUNAS VECES } & Nivel cognitivo & $\begin{array}{l}\text { Me preocupo. } \\
\text { Siento miedo. } \\
\text { - Mengo pensamientos o sentimientos negativos. } \\
\text { - Siento ganas de llorar. }\end{array}$ & $\begin{array}{l}7 \\
17 \\
13 \\
13 \\
10\end{array}$ & Primer lugar \\
\hline & Nivel fisiológico & $\begin{array}{l}\text { - El corazón me late muy rápido y/o me falta el } \\
\text { aire y la respiración es agitada. } \\
\text { - Siento molestias en el estómago. } \\
\text { - Me tiemblan las manos o las piernas. } \\
\text { Se me seca la boca y tengo dificultades para } \\
\text { trazar. }\end{array}$ & $\begin{array}{l}13 \\
8 \\
12 \\
10\end{array}$ & Segundo lugar \\
\hline & Nivel motor & $\begin{array}{l}\text { - Realizo movimientos repetitivos con alguna } \\
\text { parte de mi cuerpo, me quedo paralizado o mis } \\
\text { movimientos son torpes. } \\
\text { - Fumo, como o bebo demasiado. } \\
\text { - Me cuesta expresarme verbalmente o a veces } \\
\text { tartamudeo. }\end{array}$ & $\begin{array}{c}12 \\
3 \\
12\end{array}$ & Tercer lugar \\
\hline
\end{tabular}

Las situaciones generadoras de estrés académico planteado en los ítemes $2,4,6,10$ y 11 del instrumento, están relacionados en una forma significativa $(p<$ $0,05)$ y altamente significativa $(p<0,01)$ con los tres niveles de respuestas es decir con las manifestaciones cognitivas, fisiológicas y motoras. Las situaciones generadoras de estrés académico planteado en el item 1 está relacionado significativamente con dos niveles de respuestas es decir con las manifestaciones cognitivas y fisiológicas.Las situaciones generadoras de estrés académico planteado en los ítemes 5 y 8 están relacionados significativamente sólo con un nivel de respuesta y ésta es con la manifestación cognitiva. La situación generadora de estrés académico planteado en el ítem 9 está relacionada significativamente también con un solo nivel de respuesta y ésta es con la manifestación motora. Las situaciones generadoras de estrés académico planteado en los ítemes 3 y 7 no se relaciona con ningún nivel de respuesta es decir son situaciones generadoras de estrés pero aparentemente no generan ninguna respuesta orgánica en los estudiantes. 
Tabla 1. Correlaciones entre las situaciones generadoras de estrés académico y las manifestaciones de la respuesta por Ítem.

\begin{tabular}{clccccc}
\hline $\begin{array}{c}\text { Situación } \\
\text { generadora } \\
\text { de estrés } \\
\text { académico }\end{array}$ & \multicolumn{2}{c}{ Manifestaciones fisiológicas } & \multicolumn{2}{c}{ Manifestaciones cognitivas } & \multicolumn{2}{c}{ Manifestaciones motoras } \\
\cline { 2 - 7 } & $\mathbf{r}$ de Pearson & $\begin{array}{c}\mathbf{p} \\
\text { valor }\end{array}$ & r de Pearson & $\begin{array}{c}\mathbf{p} \\
\text { valor }\end{array}$ & r de Pearson & $\begin{array}{c}\mathbf{p} \\
\text { valor }\end{array}$ \\
\hline Item 1 & $0,434^{*}$ & 0,034 & $0,421^{*}$ & 0,040 & 0,182 & 0,394 \\
Item 2 & $0,679^{* *}$ & 0,000 & $0,595^{* *}$ & 0,002 & $0,410^{*}$ & 0,046 \\
Item 3 & 0,352 & 0,092 & 0,304 & 0,149 & 0,362 & 0,082 \\
Item 4 & $0,559^{* *}$ & 0,005 & $0,597^{* *}$ & 0,002 & $0,490^{*}$ & 0,015 \\
Item 5 & 0,319 & 0,128 & $0,497^{*}$ & 0,014 & 0,281 & 0,183 \\
Item 6 & $0,499^{*}$ & 0,013 & $0,509^{*}$ & 0,011 & $0,626^{* *}$ & 0,001 \\
Item 7 & 0,122 & 0,570 & 0,309 & 0,142 & 0,397 & 0,055 \\
Item 8 & 0,337 & 0,108 & $0,482^{*}$ & 0,017 & 0,402 & 0,051 \\
Item 9 & 0,292 & 0,167 & 0,346 & 0,097 & $0,425^{*}$ & 0,039 \\
Item 10 & $0,583^{* *}$ & 0,003 & $0,645^{* *}$ & 0,001 & $0,549^{* *}$ & 0,006 \\
Item 11 & $0,607^{* *}$ & 0,002 & $0,570^{* *}$ & 0,004 & $0,429^{*}$ & 0,036 \\
\hline
\end{tabular}

(*) La correlación es significativa al nivel de 0,05.

${ }^{(* *)}$ La correlación es significativa al nivel de 0,01 .

Indiscutiblemente el estrés afecta notoriamente el desempeño académico de los estudiantes de primero dada la notoriedad de respuesta del nivel cognitivo frente a situaciones de estrés. Así como repercute en los niveles fisiológicos y motor los que pueden deteriorar la salud si no se establecen mecanismos de afrontamiento que esté respaldado por la facultad a través de políticas de prevención y promoción de la salud mental del estudiante. La vulnerabilidad al estrés puede constituirse en elemento predictor de resultados académicos no satisfactorios en el proceso de enseñanza aprendizaje de las diferentes asignaturas del primer semestre académico de la FEN.

\section{DISCUSIÓN}

El instrumento de evaluación muestra una sensibilidad para las valoraciones tanto globales como particulares del estrés. En una fase primera el estudio abordó el análisis en forma global donde por los resultados obtenidos el inventario de estrés académico sí pone de relieve que el estrés informado por los estudiantes se repercute fundamentalmente en la respuesta de tipo cognitivo. Dicho de otra manera los alumnos, sobre todo, "piensan" de forma negativa o se preocupan ante determinadas situaciones académicas. Ahora bien, aunque habría que confirmarlo en trabajos posteriores.
Es interesante constatar cómo son las situaciones relacionadas con la sobrecarga académica y la falta de tiempo las que provocan un mayor nivel de estrés, por encima incluso de las situaciones de evaluación. Esto, creemos que es confirmatorio del llamado Modelo de Control o mejor dicho de la falta del mismo en el sentido de que la sensación de no poder abarcar todo lo que han de hacer acentuará la sensación de falta de control. El mayor nivel de estrés informado por los estudiantes de primer semestre parece apoyar, a su vez, esta falta de control en lo que tiene de revelador acerca de la no posesión de soluciones a los nuevos requerimientos que tienen que abordar por su entrada en la Universidad.

Por otro lado cada estudiante tiene particularidades individuales que va a marcar diferencias en las reacciones frente al estrés lo que se sustenta en el modelo de Lazarus complementándose con las reacciones propias biológicas y porqué no decirlo también influenciadas por factores externos como la sociedad y la misma cultura (teorías biológicas y socioculturales) que conducen al estudiante a situaciones de crisis necesarios en el proceso de crecer y aprender rodeados de muchos agentes estresores no solo académicos sino de orden también general frente a los cuáles la naturaleza los ha dotado de mecanismos compensatorios naturales que varían de persona a 
persona y que los fortalecen proporcionándoles una barrera natural, sin embargo no todos responden como se explica siendo necesario incorporar formas de afrontamiento que ayude a superar este problema.

Los datos obtenidos en el estudio realizado nos permitieron llevar a cabo un análisis de datos en una primera parte no estadística y estadística en forma genérica, dejando para la segunda fase del estudio la forma más especifica o particular es decir relación de cada ítems del primer instrumento con ítems del segundo. En la segunda fase del estudio se obtiene como resultado que la clasificación por niveles de estrés se encuentra en: alto, medio y bajo ya que un número significativo de estudiantes del total matriculados en el salón la padecen en estos niveles provocado por las situaciones generadoras de estrés descritas en orden de importancia; de las cuales fundamentalmente por su relevancia se encuentran las relacionadas con la saturación de actividades académicas y la falta de control y organización del tiempo por el estudiante, la exigencia académica por las mismas características de la carrera (formación teórica/práctica de las propias asignaturas, modalidad de desarrollo de las mismas,el tiempo para el cumplimiento de trabajos obligatorios que podría deberse a la falta de coordinación entre asignaturas para la asignación de tareas y por supuesto la natural carga de estrés que es provocada por los exámenes obligatorios a los que están sujetos los estudiantes cuyo resultado es la significativa presencia de estrés en su nivel alto y medio en los estudiantes del primer semestre académico. Por otro lado, así como hay situaciones generadoras de estrés académico para muchos estudiantes y que se categorizan en medio y alto, también hay situaciones que para un grupo de estudiantes les provocan solo algo de estrés y están relacionadas por ejemplo con situaciones que tienen relación con el contacto directo con el profesor o aquellos que tengan que ver con su participación individual $o$ en grupo e incluso para algunos estudiantes la realización de los exámenes solo les provoca algo de estrés. Finalmente también tenemos aquellas situaciones como número de estudiantes en un aula que definitivamente solo para un grupo menor de estudiantes les provocará algo de estrés, lo que da clara evidencia que las situaciones generadoras de estrés académico no tienen la misma influencia ni respuesta en los estudiantes, corroborándose así que todo ser humano es en su contexto general diferente uno de otro. Asimismo, como hay situaciones generadoras de estrés académico para muchos estudiantes y que se categorizan en alto, medio, y algo de estrés, también hay situaciones que para un grupo de estudiantes no les provoca absolutamente nada estrés. Estas situaciones están relacionadas por ejemplo con situaciones que tienen que ver con el número de estudiantes en el salón y su convivencia como compañeros de estudio, el contacto directo con el profesor o aquellos que tengan que ver con su participación individual o en grupo dentro del proceso de formación, lo que da clara evidencia que existen situaciones generadoras de estrés académico que no provocan absolutamente nada, cabe resaltar que esta afirmación solo es válida para un pequeño grupo de estudiantes. En otro espacio del análisis encontramos que la frecuencia de padecimiento referido a los ítemes de los tres niveles de respuestas del organismo del estudiante son: casi siempre, con bastante frecuencia y algunas veces; la información es importante dado que en primer lugar, sus respuestas orgánicas se vinculan con los niveles de respuesta cognitivas, fisiológicas y con respuestas motoras frente a las situaciones generadoras de estrés, en segundo lugar los estudiantes cuya frecuencia de padecimiento es casi siempre, reaccionan con respuestas frente a las situaciones generadoras de estrés preocupándose y sintiendo miedo que corresponde al nivel de respuesta cognitiva. Fisiológicamente lo más significativo es sintiendo molestias en el estómago, con temblores en las manos y piernas y teniendo dificultad para expresarse verbalmente, mientras que los estudiantes que con bastante frecuencia reaccionan con respuestas frente a las situaciones generadoras de estrés lo hacen preocupándose también, pero a diferencia del anterior se resalta la reacción relacionada a la inseguridad y a las ganas de llorar; dentro del nivel cognitivo, en lo fisiológico las molestias en el estómago con mayor importancia y en el nivel motor los movimientos repetitivos en el cuerpo y la dificultad para expresarse verbalmente. En los estudiantes que solo algunas veces reaccionan con respuestas frente a las situaciones generadoras de estrés y lo hacen en el nivel cognitivo con mayor notoriedad por los resultados obtenidos sintiendo miedo, teniendo sentimientoso pensamientos negativos, sintiéndose inseguros y con ganas de llorar; en el nivel fisiológico, el corazón les late muy rápido y/o les falta aire y la respiración es muy agitada, se les seca la boca y les tiemblan las manos y finalmente en el 
nivel motor hay movimientos repetitivos en el cuerpo y les cuesta expresarse verbalmente. Si estas reacciones se observan detenidamente podemos apreciar el nivel de atención y cuidado que se debería tener con todos los estudiantes, pero especialmente con aquellos estudiantes que ALGUNAS VECES tienen reacciones frente a las situaciones de estrés, fundamentalmente porque la manifestación de respuestas en los tres niveles que presentan podrían tener consecuencias muy negativas para la persona, por tanto deberían ser tomadas en cuenta institucionalmente por la necesidad de atención que amerita. De acuerdo a estos mismos resultados los estudiantes que reaccionan casi siempre y los que lo hacen con bastante frecuencia a las situaciones generadoras de estrés, el tipo de respuesta es menos crítica por decirlo de alguna manera que aquellos que solo algunas veces tienen reacciones cognitivas, fisiológicas y motoras ya que al parecer este grupo cuando tiene eventualmente respuestas en los tres niveles lo hace con mayor potencia siendo más crítica y dañina la situación para la persona. Respecto a la relación entre cada ítem del primer instrumento (situación generadora de estrés) con cada ítem del segundo instrumento (referido a los tres niveles de respuesta) analizada más específicamente, es como sigue: Los ítemes 2, 4, 6, 10 y 11 se relacionan con las manifestaciones cognitivas, fisiológicas y motoras de una forma significativa y altamente significativa, mientras que el ítem 1 se relaciona significativamente con las manifestaciones solo cognitivas y fisiológicas, los ítemes 5 y 8 se relacionan significativamente sólo con las manifestaciones cognitivas y el ítem 9 se relaciona únicamente con las manifestaciones motoras, finalmente los ítemes 3 y 7 no se relacionan con ninguna lo que podemos sintetizar en que si bien es cierto los 11 ítemes se comportan como situaciones generadoras de estrés por los resultados obtenidos dos de ellos no guardan relación con ninguna de las respuestas o manifestaciones frente a las situaciones de estrés por parte de los estudiantes.

Se corrobora por tanto, que las situaciones que provocan estrés académico en el $100 \%$ de estudiantes son: la falta de tiempo para poder cumplir con las actividades académicas, la realización de un examen y las exposiciones de trabajos en clase, convirtiéndose en situaciones generadoras de estrés académico prioritarias altamente estresantes en estudiantes del primer semestre de la Facultad. Los resultados no pueden extrapolarse a todos los primeros semestres de todas las facultades de la Universidad, en virtud a que el grupo de estudio pese a ser el 100 por ciento fue muy pequeño. Es unánimemente reconocido que el ingreso en la Universidad representa una transición que requiere de un periodo de adaptación más o menos prolongado quizá no estaría de más pensar no sólo en intervenciones de tipo individual sino también de tipo institucional, ya que las universidades no han puesto en marcha mecanismos de adaptación de nuevos estudiantes.

\section{LITERATURA CITADA}

Barlow y Durand 2003. Psicopatología. España, Edit Área Universitaria.

Bloomfield y Robert, B. K. 1988. Felicidad, el programa de la MT, psiquiatría e lluminación, Dr. Harold, Edit.Grijalbo.

Cruz, C; Vargas, L. 1998. Estrés, entenderlo es manejarlo. Santiago de Chile: Ediciones Universidad Católica de Chile, pp.127-134.

Fuentenebro, D. F. 1991. Psicología médica, psicopatología y psiquiatría, España, Edit.

Garfield, L. S. 1979. Psicología clínica, México, Edit. El manual moderno, S.A. de C.V. Hill, 2da. Edición, España, 1993 pp. 245-248.

McCue, J. 1982. El efecto del estrés en lo físico y su práctica. N. Eng J. Med; 306: 58- 463. McGrawHill Interamericana de España, S.A.

Michal, M. Bale. 1992. Stress. Ediciones Roche.

Rev. Psiquiatría Clínica. 1988. Estrés en estudiantes de Medicina de la Universidad Católica de Chile 25: 23-29. 